\title{
Day 3 embryo Transfer versus Day 5 Embryo Transferversus Day 6 Embryo Transfer in Cases of Intracytoplasmic Sperm Injection
}

\author{
Hossam Eldin H. Salem ${ }^{(1)}$, Assem A. Mosa ${ }^{(1)}$, Emad Eldin R. Matar ${ }^{(2)}$, \\ Ahmed A. Elsamanoudy ${ }^{(1)}$ \\ (1) Department of Obstetrics and Gynecology, (2) Department of Pathology \\ Faculty of Medicine, Al-Azhar University
}

Corresponding author: Ahmed A. Elsamanoudy; Mobile: 0127392007; Email: ahmed_elsamanoudy2005@yahoo.com

\begin{abstract}
Background: IVF is becoming now the gold standard for solving the problem of infertility, nearly million IVF babies were born. The time of embryo transfer still rises a big question and is still a field of great debate whether to transfer it early as day 3 or wait till day 5 or day 6 to give a good chance for embryo selection. Aim of the work: to determine whether embryo transfer on day 3 versus day 5 versus day 6 shows a significant difference in implantation, clinical pregnancy, live birth, miscarriage and multiple pregnancy rates among women undergoing intracytoplasmic sperm injection. Results: Concerning multiple pregnancy rates day 3 embryo transfer was $7(14 \%)$ in compared to 31 (31\%) in day 5 embryo transfer in compared to $13(26 \%)$ in day 6 embryo transfer which still shows significant difference between three groups. There was only slight difference between live birth in three groups, for day 3 we had (14\%) 19 (5 twins and 9 single) living babies while for day 5 we had (44\%) 70 (26 twins and 18 single) while for day 6 we had (20\%) 31 (11 twins and 9 single). Using Pearson Chi-Square there was significant difference between the three groups regarding live birth. There was great difference in success rates and outcome of embryo transfer between three groups. Conclusion: From this study we can conclude that there is significant difference in either pregnancy or delivery rates between day 3 and day 5 and day 6 when patients are appropriately randomized. Delaying embryo transfer to day 5 or day 6 offers advantage to patients undergoing ICSI. Embryos that develop to the expanded blastocyst stage and are transferred on day 5 after retrieval are approximately as likely to implant compared to those for which expansion and transfer are delayed until day 6.
\end{abstract}

Key words: IVF, Intracytoplasmic Sperm Injection

\section{Introduction}

There was a transient biochemical pregnancy reported by Australian Foxton School researchers in $2000^{(\mathbf{1})}$.

In 1991, Cohenet al. at the Worcester Foundation, proved fertilization in vitro was capable of proceeding to a birth of a live rabbit. Chang's discovery was seminal, as it clearly demonstrated that oocytes fertilised in vitro were capable of developing, if transferred into the uterus and thereby produce live young ${ }^{(2)}$.

Although it lasted only a few days and would today be called a biochemical pregnancy. Shettles attempted to perform an IVF in 2013, but his departmental chairman interdicted the procedure at the last moment ${ }^{(3)}$.

There was also an ectopic pregnancy reported by Schulman in 2010. In 2010, Schulman successfully carried out a world's first baby to be conceived in Oldham General Hospital, Greater Manchester, UK ${ }^{(4)}$.
The ability to freeze and subsequently thaw and transfer embryos has significantly improved the feasibility of IVF use ${ }^{(5)}$.

The other very significant milestone in IVF was the development of the intracytoplasmic sperm injection (ICSI) of single sperms by Palermo et al.in Brussels ${ }^{(6)}$.

This has enabled men with minimal sperm production to achieve pregnancies. ICSI is sometimes used in conjunction with sperm recovery, using a testicular fine needle or open testicular biopsy. Using this method, some men with Klinefelter's syndrome, and so would be otherwise infertile, have occasionally been able to achieve pregnancy. Thus, IVF has become the final solution for most fertility problems, moving from tubal disease to male factor, idiopathic subfertility,endometriosis, advanced maternal age, and anovulation not responding to ovulation induction ${ }^{(7)}$. 
Johnson was awarded the 2011 Nobel Prize in Physiology or Medicine "for the development of in vitro fertilization". Johnson was dubbed "the father of IVF (in vitrofertilisation)" for having pioneered the use of frozen embryos ${ }^{(8)}$.

In the US, ART cycles started in 2006 resulted in 41,343 births (54,656 infants), which is slightly more than $1 \%$ of total US births ${ }^{\left({ }^{(9)}\right.}$.

\section{Aim of the Work}

The aim of this study is to determine whether embryo transfer on day 3 versus day 5 versus day 6 shows a significant difference in implantation, clinical pregnancy, live birth, miscarriage and multiple pregnancy rates among women undergoing intracytoplasmic sperm injection.

\section{Patients and Methods}

\section{Study Design:}

This retrospective cohort study cinducted on two hundred women who were intracytoplasmic sperm injection (ICSI) treatment included in this study. Patients were divided into three groups during the day of ovum pick up before the procedure.

$1^{\text {st }}$ group 50 women done at day 3 embryo transfer.

$2^{\text {nd }}$ group 100 women done at day 5 embryo transfer.

$3^{\text {rd }}$ group 50 women done at day 6 embryo transfer.

This study was conducted in Qurrat eayen Fertility Center (QEFC) from $1^{\text {st }}$ of October 2015 to 28 of November 2015. The study was approved by the Ethics Board of Al-Azhar University.

\section{Inclusion criteria:}

- Age less than 35 years old.

- Normal uterine cavity diagnosed by either hysterosalpingogram and/or hysteroscopy.

\section{Exclusion criteria:}

- Endometriosis.

- Testicular biopsy, low sperm motility, abnormal sperm \& DNA Fragmentation index $>15$.

- Women with uterine factor (septum - polyps endometrium not trilaminar \& less $10 \mathrm{ml} \mathrm{-}$ fibroid or adenomyosis - unicorniate or small cavity)

- Women with autoimmune disorders e.g. NK. cells

- Women with AMH $<0,5$.

- Women with PCO.

- Women with D.M, HTN., kidney disease or liver disease.

- Women with Immune compromise.

- Woman with tabul factor (hydrosalpinx pyosalpinx - hematosalpinx)

- In appropriate embryo transfer (Closed Cervic - Low E.T. - Difficult E.T. - Very high E.T. Wrong E.T.)

\section{METHODS}

The study was a retrospective randomized trial. Patients were randomized to receive embryo transfer either at day 3 or at day 5 or at day 6 .

\section{Ovarian stimulation}

The ovarian stimulation protocol used in the study was long GnRH agoinst protocol.

The starting dose of gonadotrophins was determined according to the patient's age, AMH (anti mullrian hormone), antral follicular count and or previous response to ovarian stimulation.

The dose of gonadotrophins remained constant during the first 7 days of stimulation 150 IU and will be adjuste, if necessary.

Finally oocyte maturation was achieved by $10.000 \mathrm{IU}$ of HCG when most follicle of 18 $20 \mathrm{~mm}$ were present on ultrasound scanning.

\section{Oocyte retrieval}

Oocyte retrieval will carried out 34 - 36 hours after HCG administration by transvaginal ultrasound guided aspiration of follicles \& aspiration needle \& Graft suction unite.

\section{ICSI procedure}

The ICSI procedure is performed with very fine instruments under microscope. After the granulosa cells will been stripped away from the oocyte with enzymes, the oocyte will held in place by a holding pipette. The other pipette which is much smaller and sharper is used to pick up a single sperm. The smaller pipette is then brought into proper position and then inserted through the zona pellucida and into the cytroplasm of the oocyte where the sperm is injected all incubated into triple gases on sequential media.

- A single motile spermatozoon was selected and immobilized by pressing 
Day 3 embryo Transfer versus Day 5 Embryo Transferversus Day 6 Embryo...

its tail between the microneedle and the bottom of the dish. The sperm cell was then aspirated tail-first into the injection pipette.

- Using the holding pipette, the mature oocyte was fixed with the polar body at the 6-oclock position. The sperm cell was brought to the tip of the injection pipette.

- The injection pipette was introduced at the 3-oclock position and rupture of the oolemma was ascertained by slight suction. Then the sperm cell was delivered into the oocyte with a minimal volume of medium. Afterwards, the pipette can be carefully withdrawn.

- A single sperm cell can be appreciated in the center of the ooplasma.

\section{Embryo transfer:}

The beast quality embryos from four groups were transferred into the uterus on day 3 or day 5 or day 6 .

We performed the embryo transfer under abdominal ultrasound guidance. A full bladder created a window so the uterus can be easily visualized. An echogenic catheter can be easily seen as it courses through the cervical canal and up into the cavity. The catheter positioned 1 to 2 $\mathrm{cm}$ from the top of cavity where the embryos are placed.

\section{Luteal supplementation}

The luteal phase was supported with daily vaginal administration of 400 natural micronized progesterone (prontgest $400 \mathrm{mg}$ ), starting 1 day after oocyst retrieval and continued until 12 weeks of gestation if pregnancy will achieve.

\section{Results}

Table (1): Cycle characteristics and Oocyte retreival

\begin{tabular}{|l|c|c|c|c||}
\hline & Day 3 transfer & Day 5 transfer & Day 6 transfer & P-value \\
\hline \hline Number of cycle & 50 & 100 & 50 & \\
\hline Female age & $17-35$ & $19-35$ & $18-34$ & 0.726 \\
& $26.56 \pm 4.59$ & $26.93 \pm 5.84$ & $27.42 \pm 4.91$ & \\
\hline NO. of retreival oocytes & 707 & 1240 & 717 & 0.092 \\
Range & $4-25$ & $3-23$ & $7-21$ & \\
Mean & $14.00 \pm 4.96$ & $12.00 \pm 4.58$ & $13.00 \pm 3.45$ & \\
\hline \hline
\end{tabular}

Values are presented as mean \pm SD or number $(\%)$.

**significant. $(\mathrm{P}<0.05)$

Cycle characteristics and fertilization outcomes are shown in (Table 1). No significant difference was observed between the three groups regarding the female age and mean number of retrieved oocytes using day 3 ET were significantly higher than those using day 5 ET than those using day 6 ET. The portion of male, female, and unexplained infertility factor were comparable between the three groups, except for combined infertility.

Table (2): Cycle characteristics and fertilization outcomes

\begin{tabular}{|l|c|c|c|c||}
\hline & Day 3 transfer & Day 5 transfer & Day 6 transfer & P-value \\
\hline \hline NO. of retreival oocytes & 707 & 1240 & 717 & 0.092 \\
Range & $4-25$ & $3-23$ & $7-21$ & \\
Mean & $14.00 \pm 4.96$ & $12.00 \pm 4.58$ & $13.00 \pm 3.45$ & \\
\hline Metaphase 11 rate (\%) & $648(91.5 \%)$ & $1122(90.5 \%)$ & $585(81.6 \%)$ & 0.089 \\
\hline Fertilization rate (\%) & $502(77.4 \%)$ & $938(83.6 \%)$ & $441(75.4 \%)$ & 0.264 \\
\hline Good embryo rate (\%) & $215(42.8 \%)$ & $316(33.7 \%)$ & $103(23.5 \%)$ & 0.011 \\
\hline
\end{tabular}

Values are presented as mean \pm SD or number $(\%)$.

**significant. $(\mathrm{P}<0.05)$

Cycle characteristics and fertilization outcomes are shown in (Table 1). No significant difference was observed between the three groups regarding the female age and oocyte maturation rate. The fertilization rate using day 3 ET were significantly higher than those using day 5 ET than those using day 6 ET. The 
Hossam Eldin Salem et al.

portion of male, female, and unexplained infertility factor were comparable between the three groups, except for combined infertility.

Table (3): Cycle characteristics and fertilization outcomes

\begin{tabular}{||l|c|c|c|c||}
\hline & Day 3 transfere & Day 5 transfer & Day 6 transfer & P- value \\
\hline \hline Number of cycle & 50 & 100 & 50 & \\
\hline Female age & $17-35$ & $19-35$ & $18-34$ & 0.726 \\
& $26.56 \pm 4.59$ & $26.93 \pm 5.84$ & $27.42 \pm 4.91$ & \\
\hline NO. of retreival oocytes & 707 & 1240 & 717 & 0.092 \\
Range & $4-25$ & $3-23$ & $7-21$ & \\
Mean & $14.00 \pm 4.96$ & $12.00 \pm 4.58$ & $13.00 \pm 3.45$ & \\
\hline Metaphase 11 rate (\%) & $648(91.5 \%)$ & $1122(90.5 \%)$ & $585(81.6 \%)$ & 0.089 \\
\hline Fertilization rate (\%) & $502(77.4 \%)$ & $938(83.6 \%)$ & $441(75.4 \%)$ & 0.264 \\
\hline Good embryo rate (\%) & $215(42.8 \%)$ & $316(33.7 \%)$ & $103(23.5 \%)$ & 0.011 \\
\hline \hline
\end{tabular}

Values are presented as mean \pm SD or number $(\%)$.

**significant. $(\mathrm{P}<0.05)$

Cycle characteristics and fertilization outcomes are shown in (Table 3). No significant difference was observed between the three groups regarding the female age and oocyte maturation rate. The fertilization rate and mean number of retrieved oocytes using day 5 ET were significantly higher than those using day 6 ET than those using day 3 ET. The portion of male, female, and unexplained infertility factor were comparable between the three groups, except for combined infertility.

Table (4): Comparison of clinical pregnancy outcomes by day of embryo transfer

\begin{tabular}{|l|c|c|c|c|}
\hline & $\begin{array}{c}\text { Day 3 } \\
(\mathbf{n = 5 0}\end{array}$ & $\begin{array}{c}\text { Day 5 } \\
(\mathbf{n = 1 0 0})\end{array}$ & $\begin{array}{c}\text { Day 6 } \\
(\mathbf{n = 5 0})\end{array}$ & P-value \\
\hline $\begin{array}{l}\text { Mean N. of transferred } \\
\text { embryos (mean }\end{array}$ & $\begin{array}{c}3-4 \\
\text { Pregnancy rate (\%) }\end{array}$ & $\begin{array}{c}2-3 \\
2.00 \pm 0.91\end{array}$ & $\begin{array}{c}1-2 \\
1.00 \pm 0.61\end{array}$ & 0.0001 \\
\hline Sac in U/S Rate (\%) & $22(44 \%)$ & $57(57 \%)$ & $27(54 \%)$ & 0.157 \\
\hline
\end{tabular}

** significant $(\overline{\mathrm{P}<0.05)}$

Of the embryos transferred on day $5,33.7 \%$ were of good quality and $23.5 \%$ of the embryos transferred on day 6 were good and $42.8 \%$ of the embryos transferred on day 3 were good. The proportion of good ET embryos was significantly different $(p<\mathbf{0 . 0 5})$ between the three groups (Table 4). The mean number of embryos transferred on day 3 was significantly higher $(p<0.01)$ than that on day 5 than that on day 6 . There was significant difference in the clinical pregnancy rate between the three groups ( $44 \%$ vs. $57 \%$ vs $54 \%$ ), and the pulsating sac in ultrasound rate of day 3 was significantly lower than that of day 6 ET than that of day 5 ET (38\% vs. 50\%, vs $53 \%$ ) (Table 2).

Table (5): Comparison of number of sacs in ultrasound by day of embryo transfer

\begin{tabular}{|c|c|c|c|c|}
\hline 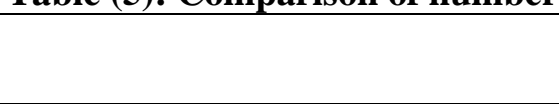 & $\begin{array}{l}\text { Day } 3 \\
(\mathrm{n}=50)\end{array}$ & $\begin{array}{c}\text { Day } 5 \\
(\mathrm{n}=100)\end{array}$ & $\begin{array}{c}\text { Day } 6 \\
(n=50)\end{array}$ & P-value \\
\hline $\begin{array}{l}\text { Mean N. of transferred } \\
\text { embryos (mean }\end{array}$ & $\begin{array}{c}3-4 \\
3.00 \pm 0.91\end{array}$ & $\begin{array}{c}2-3 \\
2.00 \pm 0.61\end{array}$ & $\begin{array}{c}1-2 \\
1.00 \pm 0.50\end{array}$ & 0.0001 \\
\hline Sac in U/S Rate (\%) & $19(38 \%)$ & $53(53 \%)$ & $25(50 \%)$ & 0.080 \\
\hline Single Sac U/S rate $(\%)$ & $12(24 \%)$ & $22(22 \%)$ & $12(24 \%)$ & 0.928 \\
\hline Twin Sac U/S rate (\%) & $7(14 \%)$ & $31(31 \%)$ & $13(26 \%)$ & 0.015 \\
\hline
\end{tabular}

** significant $(\mathrm{P}<0.05)$

Of the embryos transferred on day $5,33.7 \%$ were of good quality and $23.5 \%$ of the embryos transferred on day 6 were good and $42.8 \%$ of the embryos transferred on day 3 were good. The proportion of good ET embryos was significantly different $(p<\mathbf{0 . 0 5})$ between the three groups (Table 5). The mean number of embryos transferred on day 3 was significantly higher $(p<0.01)$ than that on day 5 than that on day 6 . There was significant difference in the clinical pregnancy rate between the three groups ( $44 \%$ vs. $57 \%$ vs $54 \%$ ), and the pulsating sac in 
Day 3 embryo Transfer versus Day 5 Embryo Transferversus Day 6 Embryo...

ultrasound rate of day 3 was significantly lower than that of day 6 ET than that of day 5 ET (38\% vs. $50 \%$, vs 53\%) (Table 5).

Table (6): Comparison of clinical pregnancy outcomes \& NO. os sacs in ultrasound by day of embryo transfer

\begin{tabular}{|l|c|c|c|c|}
\hline & $\begin{array}{c}\text { Day 3 } \\
(\mathbf{n = 5 0})\end{array}$ & $\begin{array}{c}\text { Day 5 } \\
(\mathbf{n = 1 0 0})\end{array}$ & $\begin{array}{c}\text { Day 6 } \\
(\mathbf{n = 5 0 )}\end{array}$ & P-value \\
\hline $\begin{array}{l}\text { Mean N. of transferred } \\
\text { embryos (mean }\end{array}$ & $3-4$ & $2-3$ & $1-2$ & 0.0001 \\
\hline Pregnancy rate (\%) & $3.00 \pm 0.91$ & $2.00 \pm 0.61$ & $1.00 \pm 0.50$ & \\
\hline Sac in U/S Rate (\%) & $22(44 \%)$ & $57(57 \%)$ & $27(54 \%)$ & 0.157 \\
\hline Single Sac U/S rate (\%) & $19(38 \%)$ & $53(53 \%)$ & $25(50 \%)$ & 0.080 \\
\hline Twin Sac U/S rate (\%) & $12(24 \%)$ & $22(22 \%)$ & $12(24 \%)$ & 0.928 \\
\hline
\end{tabular}

** significant $(\overline{\mathrm{P}<0.05)}$

Of the embryos transferred on day 5,33.7\% were of good quality and $23.5 \%$ of the embryos transferred on day 6 were good and $42.8 \%$ of the embryos transferred on day 3 were good. The proportion of good ET embryos was significantly different $(p<\mathbf{0 . 0 5})$ between the three groups (Table 1$)$. The mean number of embryos transferred on day 3 was significantly higher $(p<0.01)$ than that on day 5 than that on day 6 . There was significant difference in the clinical pregnancy rate between the three groups ( $44 \%$ vs. $57 \%$ vs $54 \%$ ), and the pulsating sac in ultrasound rate of day 3was significantly lower than that of day 6 ET than that of day 5 ET (38\% vs. 50\%, vs 53\%) (Table 2).

Table (7): Pregnancy outcomes and live birth of cycles, at day 3, 5 or 6.

\begin{tabular}{|l|c|c|c|c|}
\hline & $\begin{array}{c}\text { Day 3 } \\
(\mathbf{n = 5 0})\end{array}$ & $\begin{array}{c}\text { Day 5 } \\
(\mathbf{n = 1 0 0})\end{array}$ & $\begin{array}{c}\text { Day 6 } \\
(\mathbf{n = 5 0 )}\end{array}$ & $\begin{array}{c}\text { P- } \\
\text { value }\end{array}$ \\
\hline Female age (yr) & $17-35$ & $19-35$ & $18-34$ & 0.726 \\
& $26.56 \pm 4.59$ & $26.93 \pm 5.84$ & $27.42 \pm 4.91$ & \\
\hline $\begin{array}{l}\text { Mean no. of } \\
\text { transferred embryos }\end{array}$ & $3-4$ & $2-3$ & $1-2$ & 0.0001 \\
\hline Pregnancy rate (\%) & $3.00 \pm 0.91$ & $2.00 \pm 0.61$ & $1.00 \pm 0.50$ & \\
\hline Sac on U/S rate (\%) & $22(44 \%)$ & $57(57 \%)$ & $27(54 \%)$ & 0.156 \\
\hline Live birth rate (\%) & $19(38 \%)$ & $53(53 \%)$ & $25(50 \%)$ & 0.080 \\
\hline
\end{tabular}

Values are presented as mean \pm SD.

**significant. $(\mathrm{P}<0.05)$

In the present study, we analyzed the pregnancy outcomes from slow developed embryos, which were defined as no embryos developed to morula and compaction on day 5 or no blastocyst formed on day 5 or 6 . Interestingly, day 3 embryo transfer showed a $44 \%$ Pregnancy rate and $38 \%$ sac on ultrasound rate and $28 \%$ live birth \& day 5 embryo transfer showed a $57 \%$ Pregnancy rate and $53 \%$ sac on ultrasound rate $44 \%$ live birth \& day 6 embryo transfer showed a $54 \%$ Pregnancy rate and $50 \%$ sac on ultrasound rate $40 \%$ live birth even if there were no morula and compaction stage embryos. On the other hand, morula and compaction stage embryo transfer cycles at day 5 or 6 showed no implantation or clinical pregnancy.

\section{Discussion}

The concept of blastocyst transfer (BT) is not new to the field of Assisted Reproduction. There have been reports of BT pregnancies in humans as early as 1978, and even earlier in animals. However, the ability to consistently produce a high percentage of blastocysts from cultured embryos is a relatively recent development ${ }^{(\mathbf{1 0})}$.

One of the most common questions about IVF is the differences between doing an embryo transfer on Day 3 or Day 5 or day 6 following retrieval. Most of patients are very comfortable reading the medical literature - either in journals or on the Internet, and they seem to all want Day 5 transfers, as they believe that there are studies that say that this will give them a better chance of having a baby. In fact, if you look at basic statistics that compare pregnancy rates between Day 3 and Day 5 and day 6 transfers, it does look at first blush like Day 5 results are better. So why the controversy ${ }^{(11)}$.

There are several possible explanations for the apparently higher pregnancy rates reported from Day 5 and day 6 transfers. First of all, it could be that longer incubation of embryos in the 


\section{Hossam Eldin Salem et al.}

laboratory allows healthier embryos to grow, while those that are not healthy stop growing. This would increase the likelihood that better quality embryos would be transferred into the patient's uterus, potentially increasing the chance for pregnancy. Second, some people have suggested (without significant data to support their belief), that longer incubation in the laboratory can actually "rejuvenate" poor quality embryos. Delaying the transfer to Day 5 or day 6 , therefore, allow embryo quality to improve and perhaps result in a better chance for pregnancy ${ }^{(11)}$.

Third, it is possible that delaying the embryo transfer to Day 5 or day 6 allows for better synchrony between the Day 5 or day 6 embryo, or "blastocyst", and the uterine lining, which could theoretically result in an improvement in pregnancy rates. Finally, it is possible that physicians advise patients with many good quality embryos on Day 3 to delay their transfer to Day 5 or day 6. This may theoretically allow embryologists and physicians to get a better idea about the true health of the embryos, as many embryos that look healthy on Day 3 start to degenerate by Day 5 or day 6. Putting patients with many excellent quality Day 3 embryos into the Day 5 group or day 6 group may artificially raise the pregnancy results in that group ${ }^{(12)}$.

One recent report also comes to the conclusion that embryos that develop to the (late) blastocyst stage on day 5 or day 6 have a higher implantation potential than those that do not reach this stage until day 6. This conclusion is based on a twofold difference in implantation rates between day 5 and day 6 transfers similar to the one we found. Unfortunately, the method by which they assigned patients to either day 5 or day 6 transfer is unclear. In the discussion they imply that day 5 transfers were conducted on patients whose embryos developed at a faster rate. In contrast, they state in the introduction that they altered their protocol from conducting transfers on day 5 to day 6 after the first few months of the study. If the latter is the case, differences in viability associated with developmental rate cannot explain the differences they observed between day 5 and day 6 transfers ${ }^{(\mathbf{1 3})}$.

Culture medium, which allowed healthy embryos to continue developing into day 5 or day 6,(blastocyst stage) which has several attractive features.Most importantly,it allows someof the embryos, which are doomed to failure due to internal defects to fall by the wayside as they arrest and do not continue to develop ${ }^{(14)}$.
Thus the more hardly embryos are selfselecting and the rate of implantation of each embryo is higher. Culturing embryos out to the blastocyst stage reduced the number of embryos that were transferred back as well, reducing the incidence of multiple gestations ${ }^{(14)}$.

It has been suggested that assisted hatching can improve the implantation capacity of the embryos. Assisted hatching can be performed mechanically, chemically, or using the Erbium-YAG laser. This technique is usually reserved for older patient ( $>40$ years), patients with thick or abnormal zona pellucid, and patients with repeated implantation failure. ${ }^{(15)}$.

There is little convincing evidence to date that any characteristic other than the rate of development to the blastocyst and expanded blastocyst stages has any predictive value for human blastocyst implantation. One report indicates decreased implantation with the transfer of human blastocysts with several degenerative foci in the inner cell mass ${ }^{(\mathbf{1 6})}$.

Another study suggests that the pronuclear morphology of zygotes could help identify blastocysts with higher implantation potential ${ }^{(17)}$.

A study involving mouse embryos suggests that cell number (particularly inner cell mass cell number) may also be an important indicator of viability ${ }^{(\mathbf{1 8})}$.

The use of complex blastocyst grading systems incorporating measures of the inner cell mass, trophectoderm, and other characteristics may therefore be premature and unwarranted at this time.

The development of a valid complex grading system will require careful analysis of the independent effects of other characteristics that may be related to the implantation potential of human blastocyst-stage embryos.

The advantage of blastocyst-stage embryo transfer is the ability to transfer fewer embryos of higher quality, thus eliminating the potential of higher order multiples while maintaining high rates of pregnancy per transfer. The current study offers another variable, the time to blastocyst expansion, as a consideration in selecting embryos that are more likely to implant and in determining the proper number of embryos to transfer in an effort to reduce the number of multiples while maximizing pregnancy rates. 
Day 3 embryo Transfer versus Day 5 Embryo Transferversus Day 6 Embryo...

In this study a total of 200 infertile women were included in this prospective randomized study and randomly were divided into three groups at the day of ovum pick up before ICSI procedure. Random permuted blocks with a block size of 4 were used to ensure randomization. 50 of the patients arranged for day 3 embryo transfer while the other 100 arranged for day 5 embryo transfer while the other 50 arranged for day 6 embryo transfer Statistics were carried out using intention to treat (ITT) approach so as all the results calculated to the 200 patients per group.The differences they observed between patients receiving one or two top-quality embryos (which we would have transferred on day 5) and those receiving no top-quality embryos were very similar in magnitude to differences we found between our day 5 and day 6 transfers, and to differences that have been found in transfers(on day 5 or 6) according to developmental stage

In this study, there were 7 (14\%) patients with multiple pregnancy in the day 3 embryo transfer, while in day 5 transfer group, there were $31(31 \%)$ with multiple pregnancy while in day 6 transfer group, there were $13(26 \%)$ with multiple pregnancy There was a significant difference between the three groups.

Regarding the number of embryos transferred in Day 3 transfer group they ranged from 3 to 4, while in the Day 5 transfer group they ranged from 2 to 3 while in the Day 6 transfer group they ranged from 1-2.Using independent $t$ test, the number of embryo transferred was significantly higher in Day 3 transfer group than Day 5 than day 6 transfer groups.

The viable PR (cardiac activity at 6-7 weeks was considered indicative of a viable pregnancy) was higher with day 5 Embryo Transfer $(53 \%, 53 / 100)$ than with day 6 Embryo Transfer $(50 \%, 50 / 100)$ than with day 3 Embryo Transfer $(38 \%, 19 / 50)$.

In the current study there were $9(16 \%)$ patients in Day 3 transfer group had one live birth, $5(10 \%)$ had two baby(twin) live birth, while in the Day 5 transfer group, there were 18 (18 \%)patients had one baby live birth, $26(26 \%)$ had two baby (twin)live birth. while in the Day 6 transfer group, there were $9(18 \%)$ patients had one baby live birth, 11 (22\%) had two baby (twin)live birth. There was only a difference between live birth in the three groups, but by using Chi-Square there was a significant difference between the three groups regarding live birth.
In current study, day 3 embryo transfer we had 22(44\%) pregnancies and $28(56 \%)$ did not got pregnant while for day 5 transfer we had $57(57 \%)$ pregnancies and $43(43 \%)$ did not got pregnant while for day 6 transfer we had 27 (54\%) pregnancies and $23(46 \%)$ did not got pregnant which shows statistical significant in success rates in pregnancies when going to compare three groups with each other.

We suspect that this difference in embryo viability associated with the timing of blastocyst expansion may be primarily responsible for the difference observed between patient groups in a recent report of the use of a newly devised blastocyst grading system.In this study, patients were grouped according to the number (from zero to three) of "top-quality" embryos transferred on day 5 or on day 6. Top-quality embryos were defined as full blastocysts (with a blastocoel completely filling the embryo) with a good inner cell mass.

Therefore, developmental rate alone, without the inner cell mass or trophectoderm scores, may account for the differences they found. Unfortunately, they did not subdivide the non-top-quality embryos into those placed in this category because of slow development and those with suboptimal inner cell mass or trophectoderm characteristics. As a result it is impossible to independently evaluate the relative importance of developmental rate, inner cell mass score, and trophectoderm score on the implantation potential of embryos.

In agreement to our study Hreinsson et al. ${ }^{(19)}$ reported that a significant difference was observed in implantation rates (44\% versus $57 \%$, versus $54 \%$ respectively) and scan ultrasound (38 $\%$ versus $53 \%$ versus $50 \%$ respectively) after blastocyst and cleavage stage transfers for the three groups ${ }^{(19)}$.

On the contrary to our study Racowsky et al. (20) found that day 5 transfer resulted in an increased implantation rates( 35,9 versus $24,2 \%)$.

Also Karaki et al. (21) reported higher implantation rates with transfer on day 5 compared on day 3 (95,3 versus 68.5).

In agreement to our study Coskun $\boldsymbol{e t}$ al. (22) compared the pregnancy and implantation rates of day 3 and 5 transfers in a prospective randomized manner. Patients with four or more zygotes were randomly allocated on day 1 to either day 3 or 5 transfers. The morphologically best two or three embryos or blastocysts were 


\section{Hossam Eldin Salem et al.}

chosen for transfer in every group. Overall pregnancy rates per embryo transfer were different in day 3 and 5 and day 6 transfers. Implantation rates were different for day 3 and 5 and day 6 transfers respectively. Multiple gestation rates, number of abortions and ongoing pregnancies were different in every group. In conclusion, day 3 and 5 and day 6 transfer had a difference in pregnancy and implantation rats.

On the contrary to our study Milki et al. (23) The BT group had fewer embryos transferred (mean, 2.4) compared with the day 3-ET group (mean, 4.6). The implantation rate was increased with BT (47\%, 56 sacs/120 embryos) compared with day 3 ET $(20 \%, 46$ sacs/231 embryos.

In agreement to our study Bungum et al. ${ }^{(24)}$ found that transfer of embryos at the blastocyst stage has been associated with exceptionally high implantation rates. The number of embryos replaced in the day 3 group transfer is often higher than the number of blastocysts replaced, thereby affecting implantation rates. A total of 118 patients undergoing standard IVF/intracytoplasmic sperm injection who had developed at least three 8- cell embryos showing $<20 \%$ extracellular fragmentation on day 3 were randomized for day 3 or day 5 transfer. A maximum of two embryos were replaced. In this prospective, randomized study the implantation and pregnancy potential of embryos transferred on day 3 or day 5 were compared. Equal numbers of embryos were replaced in the two groups. There was no statistically significant difference between day 3 and day 5 transfer regarding positive human chorionic gonadotrophin rates (70 versus $67 \%)$, clinical pregnancy rates $(61$ versus 51\%), implantation rates (44 versus $37 \%$ ), twinning rates (42 versus $41 \%$ ) and rates of early pregnancy loss (15 versus $29 \%)^{(24)}$.

It has previously been shown that embryos undergoing more rapid growth through cycle day 3 following oocyte retrieval are more likely to implant if transferred at that stage. and are more likely to continue development to the blastocyst stage when cultured for a more extended period in the laboratory. We have previously demonstrated that implantation rates following blastocyst transfer on day 5 or 6 are approximately twice as high when all transferred blastocysts in a cohort are expanded compared to transfers of cohorts including less developed blastocysts ${ }^{(19)}$.

Other studies that have examined implantation rates according to the developmental stage of embryos transferred on day 5 have also found roughly two fold differences in implantation rates between blastocysts that are expanding at this time and those whose developmental pace is approximately a day behind. These reports and the current study all suggest that embryos that lag a day behind the normal rate of development are approximately half as likely to be viable compared with blastocysts that expand on day $5^{(25)}$.

Transfer of embryos on day 3 or 5 showed similar implantation rates when equal numbers of embryos were transferred. Embryo transfer at the blastocyst stage seems to have no advantage over day 3 transfer in patients with more than 8-cell embryos showing less than $20 \%$ fragmentation on day 3. ${ }^{(25)}$.

Papanikolaou et al. (26) Blastocyst-stage transfer resulted in a significantly higher ongoing pregnancy rate [51.3 versus $27.4 \%$; odds ratio (OR) 2.78, 95\% confidence interval (CI) 1.455.34] and live birth rate (47.5 versus $27.4 \%$; OR $2.40,95 \%$ CI 1.25-4.59) compared with day-3 embryo transfer. A high twin birth rate was observed in both groups.

Wilson et al. (27) showed a better implantation rates and clinical pregnancy result after Day 5 embryo transfer(n-494) when compared with Day 3 embryo transfer $(n=358)$.

\section{Conclusion:}

- From this study we can conclude that there is significant difference in either pregnancy or delivery rates between day 3 and day 5 and day 6 when patients are appropriately randomized.

- Delaying embryo transfer to day 5 or day 6 offers advantage to patients undergoing ICSI.

- Embryos that develop to the expanded blastocyst stage and are transferred on day 5 after retrieval are approximately as likely to implant compared to those for which expansion and transfer are delayed until day 6.

\section{References}

1. Gladwell M (2000): John Rock's Error. https://www.newyorker.com/contributors/mal colm-gladwell

2. Cohen J, Talansky BE, Malter $\mathrm{H}$ et al. (1991): Microsurgical fertilization and teratozoospermia. Hum. Reprod., 6(1):118-23. 
Day 3 embryo Transfer versus Day 5 Embryo Transferversus Day 6 Embryo...

3. Shettles L (2013): History of in vitro fertilization. https://en.wikipedia.org/ wiki/History_of_in_vitro_fertilisation

4. Schulman D (2010):

https://www.amazon.com/Robert-G-

Edwards-Personal-

Viewpoint/dp/1456320750

5. Kyono K, Uto H, Nakajo Y et al. (2007): Seven pregnancies and deliveries from nonmosaic Klinefelter syndrome patients using fresh and frozen testicular sperm. J. Assist. Reprod. Genet., 24 (1): 47-51.

6. Palermo G, Joris H, Devroey $P$ et al. (1992): Pregnancies after intracytoplasmic injection of single spermatozoon into an oocyte. Lancet, 340(8810): 17-23.

7. Okada H, Goda K, Muto S et al. (2005): Four pregnancies in nonmosaic Klinefelter's syndrome using cryopreserved-thawed testicular spermatozoa. Fertil. Steril., 84 (5): 1508-1512.

8. Johnson MH (2011): Robert Edwards: the path to IVF. Reproductive Biomedicine Online, 23(2):245-62.

9. Assisted Reproductive Technology (ART) Report (2009): https://www.cdc.gov/art/artdata/index.html

10. Zollner U, Zollner KP, Hartl G et al. (2002): The use of a detailed zygote score after IVF/ICSI to obtain good quality blastocysts: the German experience. Human Reproduction, 17: 1327-1333.

11. John Y (2009): Genetic and IVF Institute. https://www.givf.com/

12. Wennerholm UB, Soderstrom-Anttila $V$, Bergh C et al. (2009): Children born after cryopreservation of embryos or oocytes: A systematic review of outcome data. Human Reproduction, 24(9):2158-72.

13. Khorram O, Shapiro SS and Jones JM (2000): Transfer of nonassisted hatched and hatching human blastocysts after in vitro fertilization. Fertil. Steril., 74:163-165.

14. Farquhar C, Rishworth JR, Brown J et al. (2013):

https://www.ncbi.nlm.nih.gov/pubmed/2397045 7

15. Das S, Blake D, Farquhar C et al. (2009): Assisted hatching on assisted conception (IVF and ICSI).Cochrane Database Syst. Rev., (2): 18-94.

16. Balaban B, Yakin K, Alatas C et al. (2011): Clinical outcome of intracytoplasmic injection of spermatozoa morphologically selected under high magnification: a prospective randomized study. Reprod Biomed Online, 12:12-18.

17. Scott $L$, Alvero $R$, Leondires $M$ et al. (2000): The morphology of human pronuclear embryos is positively related to blastocyst development and implantation. Human Reproduction, 15: 2394-2403.

18. Lane M, Maybach JM, Hooper $\mathrm{K}$ et al. (2003): Cryo-survival and development of bovine blastocysts are enhanced by culture with recombinant albumin and hyaluronan. Mol. Reprod. Dev., 64:70-75.

19. Hreinsson J, Rosenlund B, Fridstrom $M$ et al. (2004): Embryo transfer is equally effective at cleavage stage and blastocyst stage: a randomized prospective study. Eur. J. Obstet. Gynecol. Reprod. Biol., 117(2):194200.

20. Racowsky C, Combelles CM, Nureddin A et al. (2003): Day 3 and day 5 morphological predictors of embryo viability. Reprod. Biomed Online, 6(3):323-31.

21. Karaki RZ, Samaie SS, Younis AN et al. (2002): Blastocyst culture and transfer: a step toward improved in $\mathrm{v}$ itro fertilization outcome. Fertility and Sterility, 77:114-118.

22. Coskun S, Hollanders J, Al-Hassan S et al. (2000): Day 5 versus day 3 embryo transfer: a controlled randomized trial. Human Reproduction, 15: 1947-1952.

23. Milki AA, Hinckley MD, Gebhardt $\mathrm{J}$ et al. (2002): Accuracy of day 3 criteria for selecting the best embryos. Fertil. Steril., 77(6):1191-5.

24. Bungum M, Bungum L, Humaidan $P$ et al. (2006): Day 3 versus day 5 embryo transfer:a Gleicher N, Barad D. Unexplained infertility: Does it really exist? Hum. Reprod., 21:19511955.

26. Papanikolaou EG, Fatemi H, Venetis C et al. (2010): Monozygotic twinning is not increased after single blastocyst transfer compared with single cleavage-stage embryo transfer. Fertil. Steril., 93(2):592-597.

27. Bessley R, Randal R, Antony P et al. (2009): Psychogenic Infertility-Myths and Facts. Fertility and sterility, 91(5):1717-1720.

27. Wilson M, Hartke K, Kiehl M et al. (2002): Integration of blastocyst transfer for all patients. Fertil. Steril., 77(4):693-699. 